\title{
Beam Deceleration Leads to Superior Image Quality of Biological Samples Analyzed in the Scanning Electron Microscope
}

\section{Bernd Zechmann ${ }^{1}$}

${ }^{1 .}$ Baylor University, Center for Microscopy and Imaging, Waco, TX.

Non-conductive samples such as biological specimen have to be covered with a fine layer of conductive material such as metals (palladium, iridium, gold) or carbon before they can be analyzed in the scanning electron microscope (SEM) under high vacuum conditions. If left uncoated electrons accumulate at the surface of the sample and lead to artifacts which are commonly referred to as charging [1-4]. Excessive charging leads to shifted line scans and distorted ultrastructure. Such artifacts can be avoided by imaging non-conductive samples at low/variable pressure. Under these conditions charging is neutralized by ionized residual gas molecules in the chamber [5,6]. Alternatively, non-conductive samples can also be analyzed at high vacuum conditions with beam deceleration. When using beam deceleration the stage/sample is kept at a negative bias voltage. Electrons are decelerated by this negative voltage before they reach the surface. While this approach has been extensively tested in material sciences $[6,7,8]$ very little information is available on how this method advances imaging capabilities of biological samples in the SEM. The aim of this study was to compare the ultrastructure of butterfly wings of Agraulis $s p$. and leaves of Nicotiana tabacum observed under low and high vacuum, with and without beam deceleration.

Charging resulting in shifted line scans and distorted ultrastructure was commonly observed when samples were imaged at high vacuum conditions without beam deceleration (Figure 1 A \& B). Imaging these samples at low/variable pressure conditions strongly reduced artifacts induced by charging. Nevertheless, under these conditions images appeared grainy and resolution was strongly reduced making it difficult to image fine structural details at high magnification (Figure $1 \mathrm{C} \& \mathrm{D}$ ). The application of beam deceleration significantly improved image quality of both samples when imaged at high vacuum conditions. Charging did not appear under these conditions and fine structural details on the scales of the butterfly wing and the surface of the leaf were clearly visible (Figure $1 \mathrm{E} \mathrm{\&} \mathrm{F).}$

In conclusion, the application of beam deceleration resulted in superior image quality in comparison to samples that were imaged at low or high vacuum conditions without beam deceleration. Artifacts such as charging could not be observed when beam deceleration was used.

\section{References:}

[1] J Cazaux, Scanning 26 (2004), p. 181.

[2] KH Kim et al, Materials Transactions 5 (2010), p. 1080.

[3] KS Sim, J. Microscopy 238 (2010), p. 44.

[4] R Flatabo et al, J. Microscopy 265 (2017), p. 287.

[5] B James, Trends Food Science \& Technology 20 (2009), p. 114.

[6] H Miyazaki, Pathology - Research and Practice 208 (2012), p. 503.

[7] D Phifer, Microscopy Today 17 (2009), p. 40.

[8] Q Wan, Ultramicroscopy 171 (2016), p. 126. 


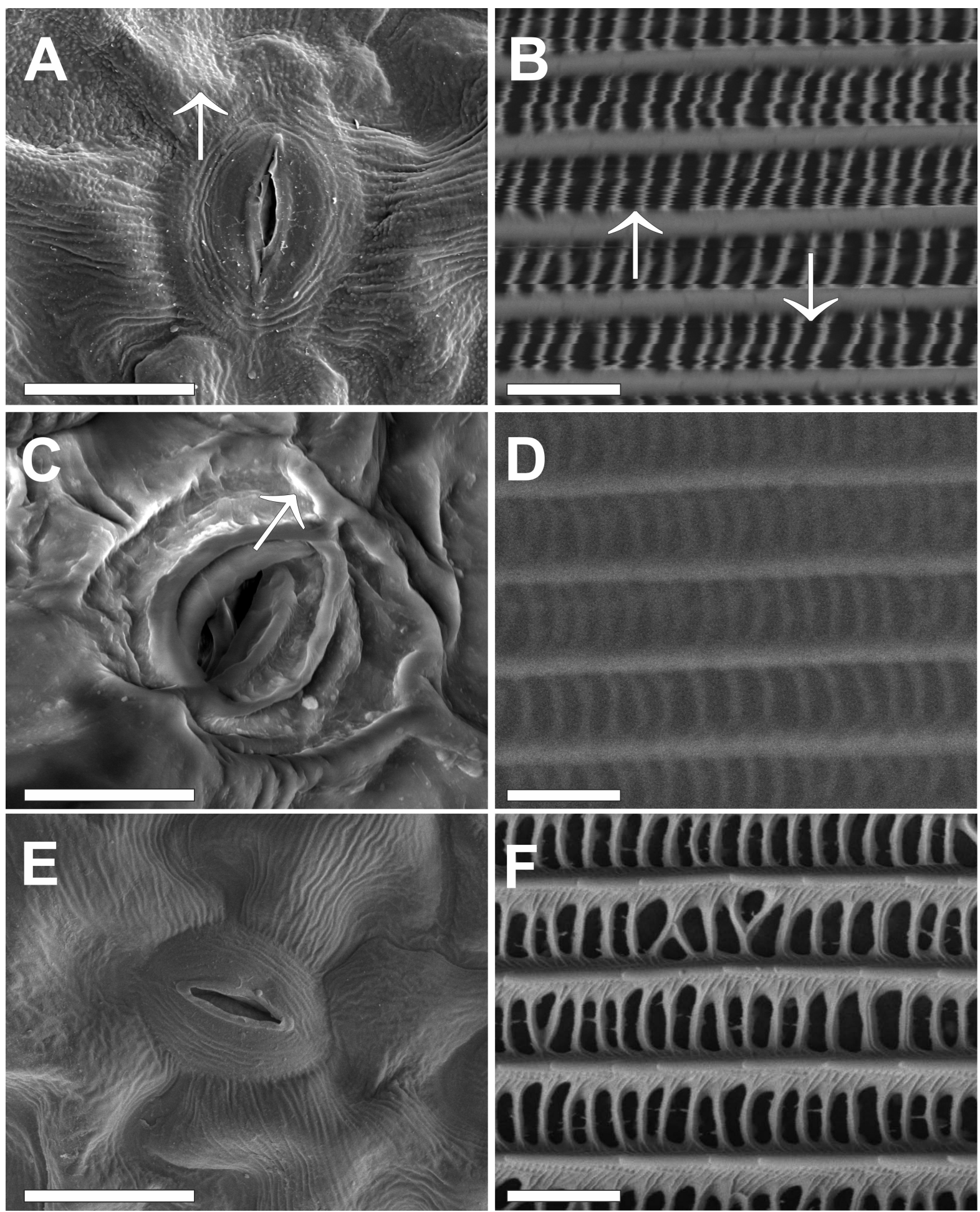

Figure 1. SEM-micrographs of butterfly wings of Agraulis sp. and stomata cells of Nicotiana tabacum under A, B) high vacuum conditions ( $3 \mathrm{kV}$ for Agraulis and $5 \mathrm{kV}$ for Nicotiana), C, D) low vacuum conditions (30 kV for Agraulis and 5kV for Nicotiana), and E, F) high vacuum conditions with beam deceleration ( $5 \mathrm{kV}$ and $-2 \mathrm{kV}$ beam deceleration). Bars $=20 \mu \mathrm{m}$ in $\mathrm{A}, \mathrm{C}, \mathrm{E}$ and $5 \mu \mathrm{m}$ in $\mathrm{B}, \mathrm{D}, \mathrm{F}$. 